\title{
Fractal Properties of Microplasma Breakdown and Structural Heterogeneity of Semiconductor Materials
}

\author{
Samuil Khanin \\ Herzen State Pedagogical University of \\ RussiaSt.Petersburg, Russia \\ antodinik@gmail.com
}

\author{
Antonina Shashkina \\ St.Petersburg State University of Film \\ and Television \\ St.Petersburg, Russia \\ shashkinaas@yandex.ru
}

\begin{abstract}
This work presents experimental results on the study of current-voltage characteristics and oscillograms of microplasma pulses of the p-n-junction avalanche breakdown. Based on the latter, the pulse duration distributions are determined. As a result, it is shown that microplasma noise has fractal properties. The latter form the basis of avalanche breakdown types developed classification. The correlation between the fractal dimension of microplasma noise and structural inhomogeneities of functional semiconductor structures is revealed.
\end{abstract}

Keywords-avalanche breakdown, microplasma, p-njunction, fractal, structural defect, semiconductor structure.

\section{INTRODUCTION}

Avalanche breakdown of real semiconductor structures is strongly localized and has microplasmic character [1, 2]. Breakdown localization usually occurs in places of various defects. These may be vacancies, dislocations, inclusions of another phase, inhomogeneities in the distribution of alloying impurities, amphoteric impurities. Dislocations [1] are the most significant here. Close to dislocation there are areas of compression and expansion of crystal lattice, which effects the width of band gap. Moreover, dislocations are centers of impurity association, and they create enhanced diffusion channels for the latter. Accordingly, the probability that carrier generating avalanche will appear in defect area is higher than for defect-free area. The concentration of charge carriers in enhanced diffusion channel is higher, and the effective thickness of depleted layer is respectively lower. As a result, the field strength locally increases, breakdown voltage decreases, microplasmas are formed. Current flowing through microplasma channel is short pulses appearing and disappearing in random times [1,2]. In the previous works [2-4] we obtained first indications that temporal distribution of microplasma pulses is random, and at the same time has self-similarity property. The discovered properties require additional studies, including those aimed at identifying correlation between the observed time distributions in the context of fractal approach and structural inhomogeneities of semiconductor materials, which is the subject of this work.

\section{MATERIALS AND METHODS}

As a follow-up to the works [2-4] we investigated gallium phosphide $p-n$-junctions used particularly in LEDs with red emission АЛ102AM (GaP:ZnO). Semiconductor structures were supplied with constant reverse voltage (supply voltage) from 10 to $19 \mathrm{~V}$. Signal received at the resistance in series connection with $p$ - $n$-junction was amplified and fed to analog-to-digital converter (ADC). Further processing of signal from ADC was carried out using Matlab mathematical package.

Supply voltage was controlled through programming, with spacing increment of about 0.005 $\mathrm{V}$ until first microplasma appeared. After that, the rate of tension growth decreased, though it was increased until next microplasma appeared. The process continued until reverse current pulses disappeared and its changes started being monotonous, i.e. semiconductor switched to developed avalanche breakdown mode [4, 5]. At the same time, the signal was recorded in the computer memory and displayed in real time on the screen in Matlab window. The proposed method was applied to 300 semiconductor structures.

The obtained quantitative data were used to construct inverse branch of current-voltage curve (I-V curve) for each studied sample, showing breaks and fractures associated with avalanche current unstable nature. With specially developed software for each point of I-V curve, it was possible to record and characterize the oscillogram of pulse current - dependence of voltage on time, with voltage dropping with resistance connected in series with $p$ - $n$-junction.

The obtained oscillograms confirmed self-similar nature of avalanche current pulses set through reverse biased $p-n$-junction. Fractal dimension of different semiconductor structures at different breakdown voltages was determined.

Algorithm for calculating fractal dimension D using Hausdorff's formula was implemented in Matlab [6]: 


$$
D=\lim _{d T \rightarrow 0} \frac{\ln n}{\ln (1 / d t)},
$$

where $d t$ is the length of the extracted segment of the investigated time series, $n$ is the number of iterations. Using wavelet analysis [7], local extrema moments of signal values along $\mathrm{Y}$ axis were determined. As a result we obtained a number of extrema values and a number of corresponding time moments (number of points on $\mathrm{X}$ axis). At the first iteration we selected a segment equal to half the length of the investigated time series. Its beginning was combined with the first point of the series. The segment "overlapped" a number of points. Then the beginning of the segment was combined with next nearest nonoverlapped point. Then we determined the number of overlaps. At the second iteration: we divided the segment in half, and counted number of series overlaps similarly. Then we did $i$ number of steps. As a result, we got dependence of number of overlaps $n$ on length of used segment $d t$, and plotted dependence $\ln (n)$ against $\ln (1)$ dt) (Fig.1). Using least square method we made a trend line and defined tangent of its inclination angle, which is equal to $D$. Meanwhile we established that the value of fractal dimension significantly depends on reverse voltage supplied to a sample.

To obtain additional information on the structure of semiconductor structures we recorded and analyzed $\mathrm{I}-\mathrm{V}$ curve direct branches.

\section{RESULTS AND DISCUSSION}

Fig. 2-4 represent indicative oscillograms of pulse current, analysing which we can classify avalanche breakdown by fractal properties. We can distinguish the following types of avalanche breakdown:

Type B (basic) is characterized by long current pulses of same amplitude, fractal properties are distinct (dimension is usually up to $0,6-0,7$ ) (Fig. 2).

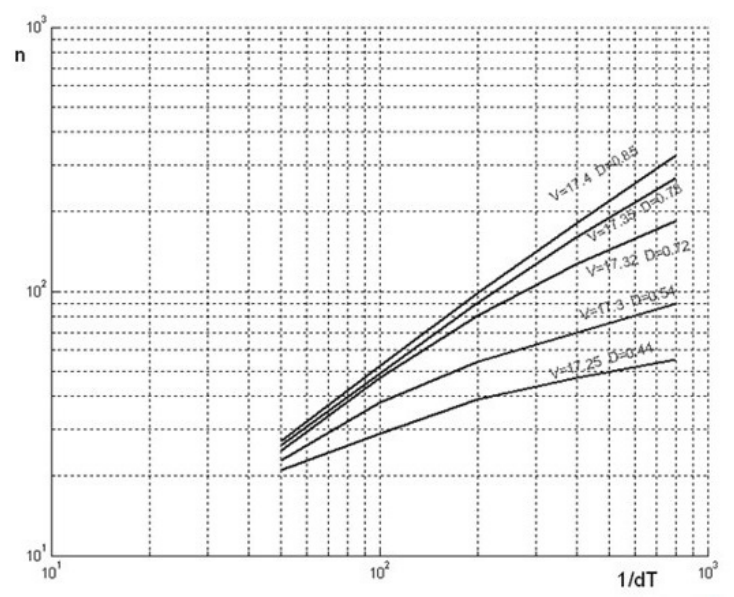

Fig. 1. Fractal dimension curves

Type $\mathrm{C}$ (chaotic) is characterized by chaotic change in duration and amplitude of microplasma noise (absence of significant pulses) and weaker fractal properties (fractal dimension is close to 1) compared to type B (Fig. 3).
Type $\mathrm{S}$ (secondary) is close to type $\mathrm{B}$, though pulses are very short, numerical value of fractal dimension is intermediate between values of fractal dimensions of type C and type B (Fig. 4). One may note that type B breakdown can regenerate into type S breakdown. Such samples are noticed to have increased series resistance.

Materials science, condensed matter physics and other sciences have general hierarchy of real structures: macrostructure, mesostructure, microstructure [8]. At all three levels, with load on a sample, there are processes of self-organization in structure. Emergence and resorption of microplasmas is a process that takes place at microlevel, and it can be regarded as fluctuations between two stationary states. These fluctuations are determined by changes in parameters at microlevel, which depend on macroparameters, most important of which is temperature [9-11]. Therefore, presence of fractal properties (selfsimilarity properties) and multi-level amplitude of current pulses indicate that in avalanche breakdown several competing factors act simultaneously, and they must be considered together in analysis of avalanche breakdown mechanisms of $p$ - $n$-junction. These factors are: dependence of concentration, mobility of charge carriers, mean free path on temperature and field value.

Feedbacks common to self-organizing processes were reflected in the developed simulation mathematical model. Within the model processes taking place in $p$ - $n$-junction were presented as dynamic positive (contributing to development of breakdown) and negative (suppressing breakdown) feedbacks, which allowed to take into account the most important factors affecting avalanche formation during microplasma noise analysis. The theoretical values of fractal dimension calculated using the model showed good correspondence to the values obtained during the experiment. Thus, we verified the classification.

Let us turn to the main goal of this work - establishing correlation between fractal dimension of microplasma noise and structural inhomogeneities of semiconductor structures. As is known, defectiveness degree of structure can be judged by analysis of $\mathrm{I}-\mathrm{V}$ curve direct branch [12]. Excess currents (in our case above $10^{-12} \mathrm{~A}$ ) on it with displacements less than $1 \mathrm{~V}$ are associated with tunneling, which can occur in the area of extended defects (dislocations, packaging defects, etc.) and local heterogeneities of composition. In this case, the coefficient of nonideality (which is part of the Shockley equation for the diode) is much more than two [12]. 


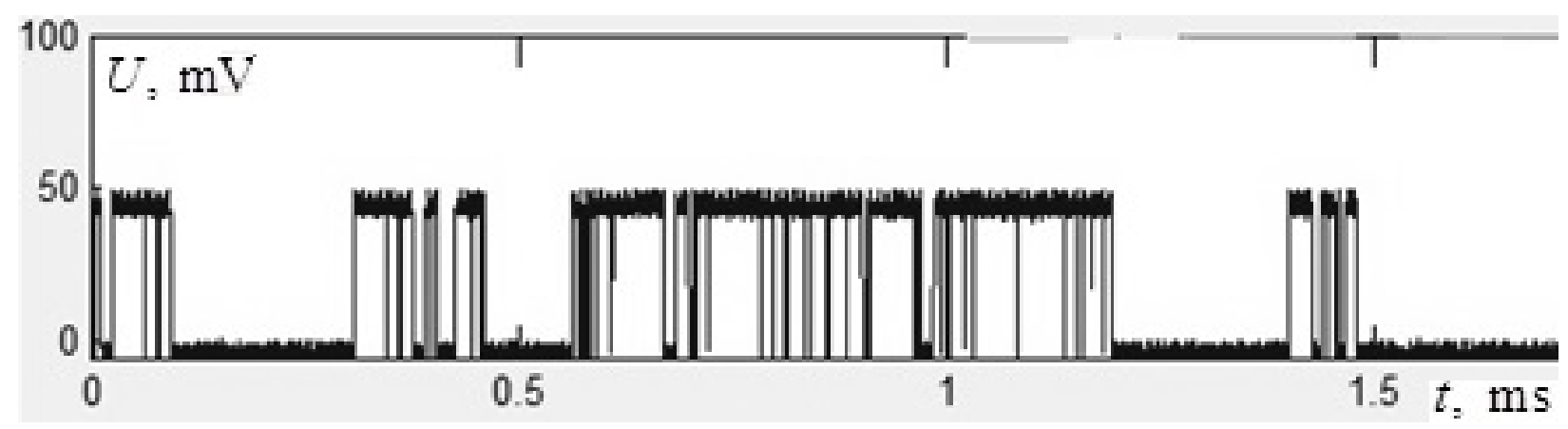

Fig. 2. Oscillogram of pulse current through reverse biased $p-n$-junction at avalanche breakdown type B

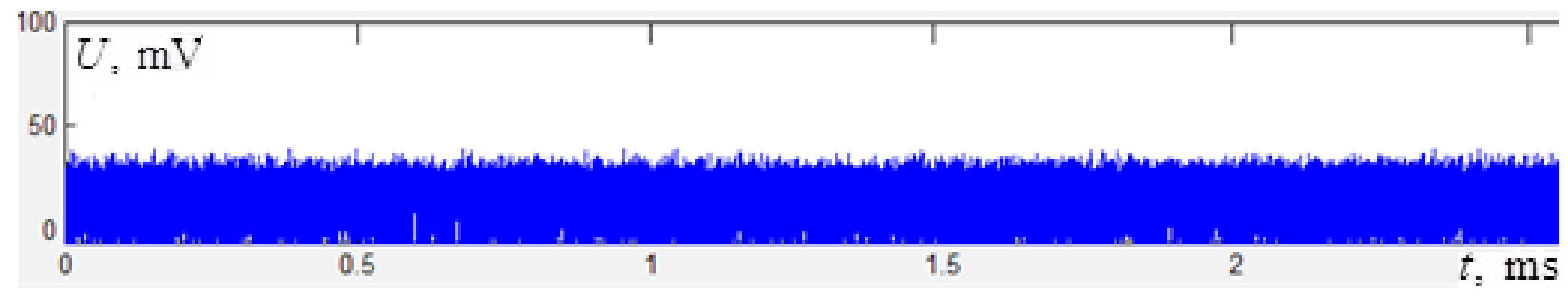

Fig. 3. Oscillogram of pulse current through reverse biased $p$ - $n$-junction at avalanche breakdown type $\mathrm{O}$

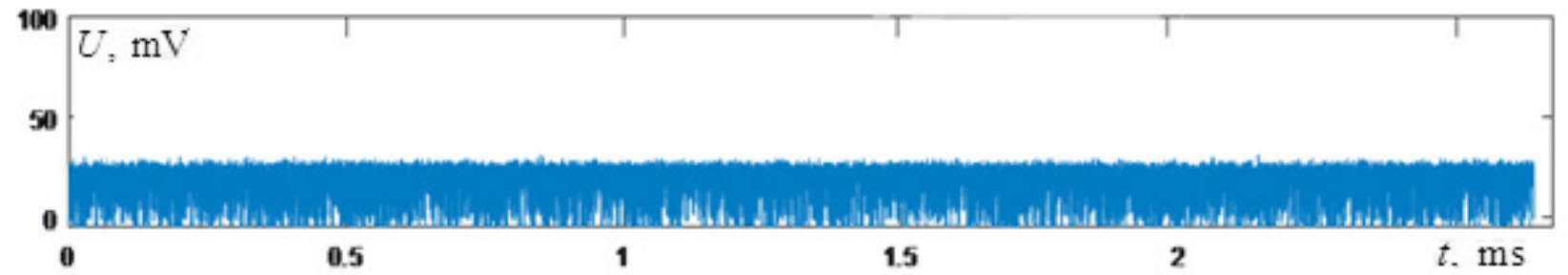

Fig. 4. Oscillogram of pulse current through reverse biased $p$ - $n$-junction at avalanche breakdown type A

As the experiment shows, most of the type B devices have tunnel component at displacements less than $1 \mathrm{~V}$ on I-V curve straight branch (Fig. 5), on type $\mathrm{C}$ and $\mathrm{S}$ structures such components are practically not detected in the same voltage range (Fig. 6).

We defined the value of direct current at which yellow glowing appears in LEDs based on the studied structures. According to the results of these studies we found that most LEDs made on the basis of group B structures begin to emit in yellow spectrum at current values close to $120 \mathrm{~mA}$, whereas for LEDs of group S and C glow appears at current values above $200 \mathrm{~mA}$. This may be due to a large number of defects that contribute to formation of microplasmas in avalanche breakdown. Traditional methods cannot effectively detect such defects.

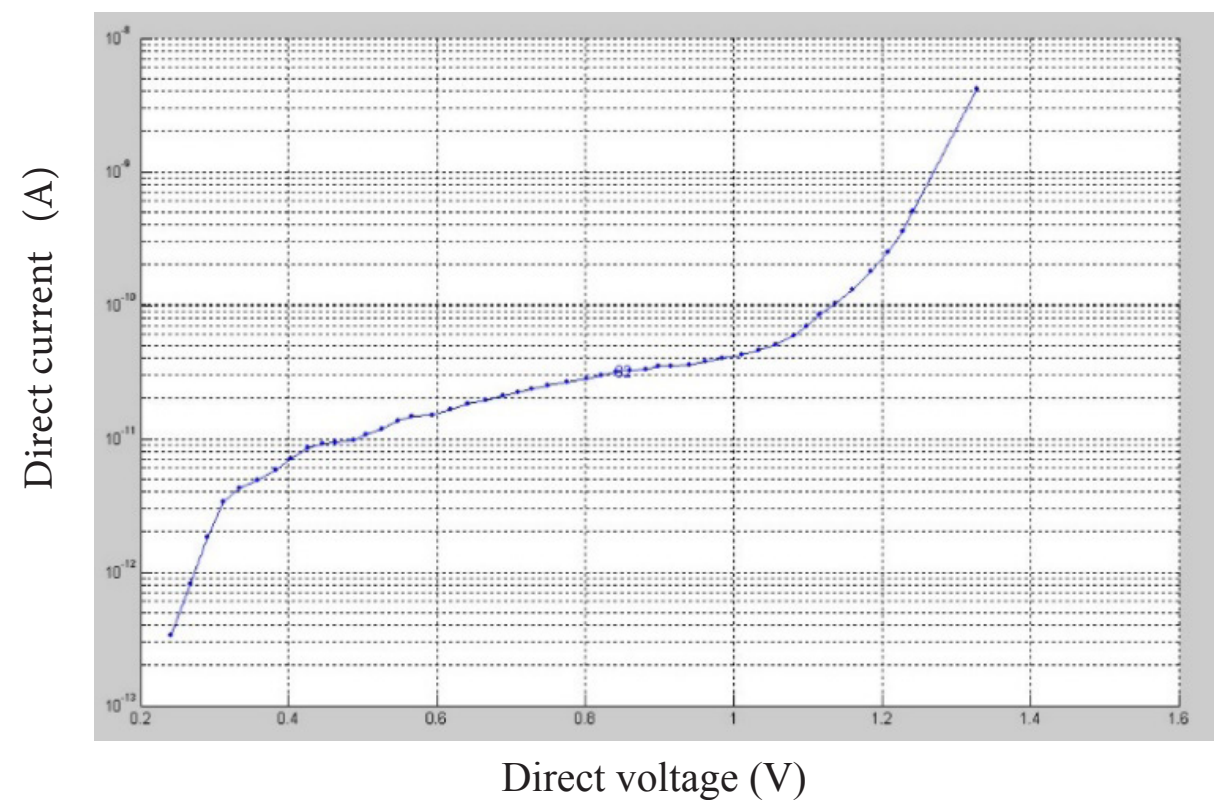

Fig. 5. I-V curve direct branch at avalanche breakdown type B 


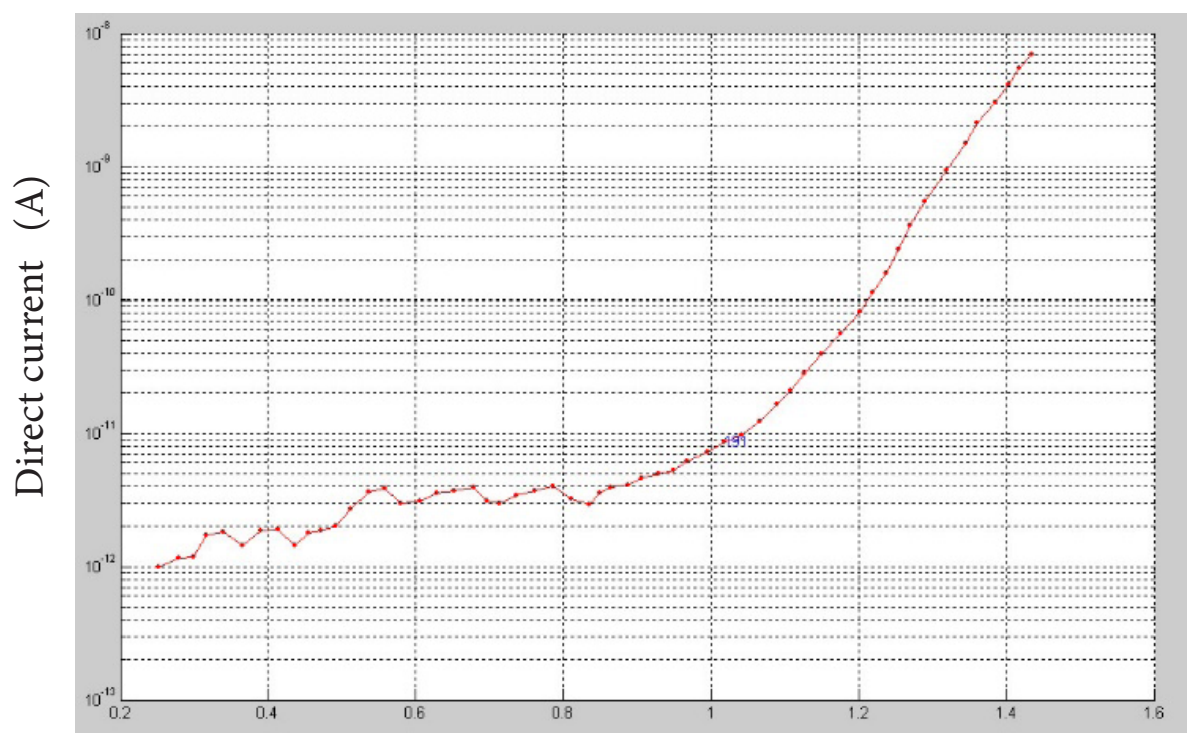

Direct voltage (V)

Fig. 6. I-V curve direct branch at avalanche breakdown type $\mathrm{S}$

Summarizing the above, we can conclude that the applied experimental method for analysis of fractal properties can be used as a basis for nondestructive control method of structural inhomogeneities in development and production of semiconductor devices.

\section{CONCLUSIONS}

Our analysis showed that microplasma noise has fractal properties. Based on the latter we developed an avalanche breakdown types classification. The obtained data confirm that the semiconductor structure sample belongs to one or another classification group. Thus, we found that strongly pronounced fractal properties may indicate the presence of a large number of defects in semiconductor structure. Based on this we propose nondestructive control method for semiconductor devices.

\section{REFERENCES}

1. Grekhov I.V., Serezhkin U.N. Avalanche breakdown of p-n-junction in semiconductors [Text]. - L.: Energia, Leningrad department, 1980. p.152

2. Vorotkov M.V., Skvortsov N.N., Shashkina A.S. Fractal properties of microplasma noise // Innovative technologies in media education. Materials of III All-Russia research and practical conference, March 27-28, 2015. Issue 3. SPb: SPbGUKIT, 2015. Pp. 65-71.

3. Shashkina A.S., Krivosheikin A.V., Skvortsov N.N., Vorotkov M.V. Fractal properties of avalanche breakdown of LED // STB SPbPU. Physicomathematical sciences. 2016. №4 (253) 2016. Pp. 85-93.

4. Shashkina A.S., Krivosheikin A.V., Skvortsov N.N., Vorotkov M.V. Avalanche breakdown of p-n-junction in radiotechnics // Scientific and technical bulletin for information technology, mechanics and optics. 2016. Vol. 16. № 5. Pp. 864-871.

5. Ivanov P.A., Potapov A.S., Samsonova T.P., Grekhov I.V. Currentvoltage characteristics of high-voltage $4 \mathrm{H}-\mathrm{SiC} \mathrm{p}^{+}-\mathrm{n}_{0}-\mathrm{n}^{+}$diodes in the avalanche breakdown mode// Semiconductors. 2017. T. 51. № 3. Pp. 374-378.

6. Feder E. Fractals. - M: MIR, 1991. - Pp. 254

7. Shchitov I.N., Galkina V.G. Introduction in signal theory. Part 3. Wavelet transform. SPb: SPbGUKIT publishing house, 2012. p.99

8. Tushinskiy L.I. Synergetical basis for structures classification and evolution in modern material sciences // Fractals and applied synergetics 2005: Collection of articles. - M: Interkontakt-Nauka, 2005.

9. Schoell, E. Self-organisation in semiconductors. Nonequillibrium phase junctions in semiconductors due to generation-recombination processes. / E. Schoell - M.: Mir, 1991. - p.464

10. Kuznetsov V. S., Kuznetsov P. A. To the question of spatial self-organisation of current free carrier in strong electric fields // Materials for electronic technology. 2012. №3.

11. Skvortsov N.N., Shashkina A.S. Avalanche breakdown self-organisation in LEDs // Papers of VII All-Russia conference «Irreversible processes in nature and technics». BMSTU, January, 27-29 Moscow, 2015. Part II, pp. 46-48.

12. W. Shockley, W.T. Read. Phys. Rev., 87, 835 (1952) 